Amini, F. Production Method for Violent TCB Jet Plasma from Cavity. in The 12th International

Conference on Condensed Matter Nuclear Science. 2005. Yokohama, Japan.

\title{
Production Method for Violent TCB Jet Plasma from Cavity
}

\author{
Farzan Amini \\ Department of Mechanical Engineering ,Farab Compny \\ No.30, Mirhadi St. Vali-e-Asr Ave. Tehran - Iran \\ farzanamini@yahoo.com
}

\section{Introduction}

One of our hydropower plants (Masjed - E - Soleyman, Iran) has four Francis turbines. Two units on the right side (looking downstream) share a common penstock and a common long tailrace tunnel, and the other two units on the left also share a penstock and tailrace tunnel.

Upon commissioning of one unit, the hydraulic transient in the draft tube during load rejection above $75 \%$ was excessive. It was apparent that the guide vane closing law that had been adopted would result in water column separation during load rejection at full power. Tests with a slower closing rate showed that the risk of column separation was reduced, but a violent surge developed in the draft tube close to maximum over speed.

The energy level and cavity volume that are produced are much more than those of regular TCB (Transient Cavitation Bubbles) experiments, and therefore, we should expect more intense effects than a regular TCB jet produces.

\section{Load Rejection Test}

When the generator is disconnected from its load, the rotational speed increases due to the hydraulic torque on the turbine. The governor senses the higher speed and causes the turbine distributor to close rapidly to prevent the rotational speed from reaching excessive values. The rapid closure of the distributor causes the spiral case pressure to rise and the draft tube pressure to drop as a result of water hammer effects.

The risks during load rejection include: excessive pressure in the penstock; an excessive pressure drop in the draft tube; and an excessive rotational speed inducing shaft vibrations and loss of bearing oil.

Water hammer calculations were done to see if better break point and closing rates could be found. They indicated that load rejection at full power could be safe with a slower first closing stroke and a lower break point. Tests with the new settings caused a sharp pressure surge in the draft tube (Figure 1). This surge propagated to the spiral case and penstock and caused big dynamic loads on the mechanical assemblies of the turbines. It is due to a sudden collapse of the draft tube vortex cavity or to a burst of self-excited instability at the particular unsteady operating conditions of the unit. These unsteady conditions typically occur at around 50\% of best efficiency energy coefficient, $30 \%$ of 
best efficiency discharge coefficient, and a low cavitation coefficient. The initial conditions of test and results of load rejection are as follows:

\section{Initial Conditions of Test}

Head water level:

$367.7 \mathrm{~m}$

Tail water level:

$222.4 \mathrm{~m}$

Opening before GCB off $\quad 86.7 \%$

Power before GCB off

Test Results:

Max. speed rise: $\quad 150.3 \%$

Min Draft tube pressure: 0.03 bar

Max. Draft tube pressure: 7.01 bar
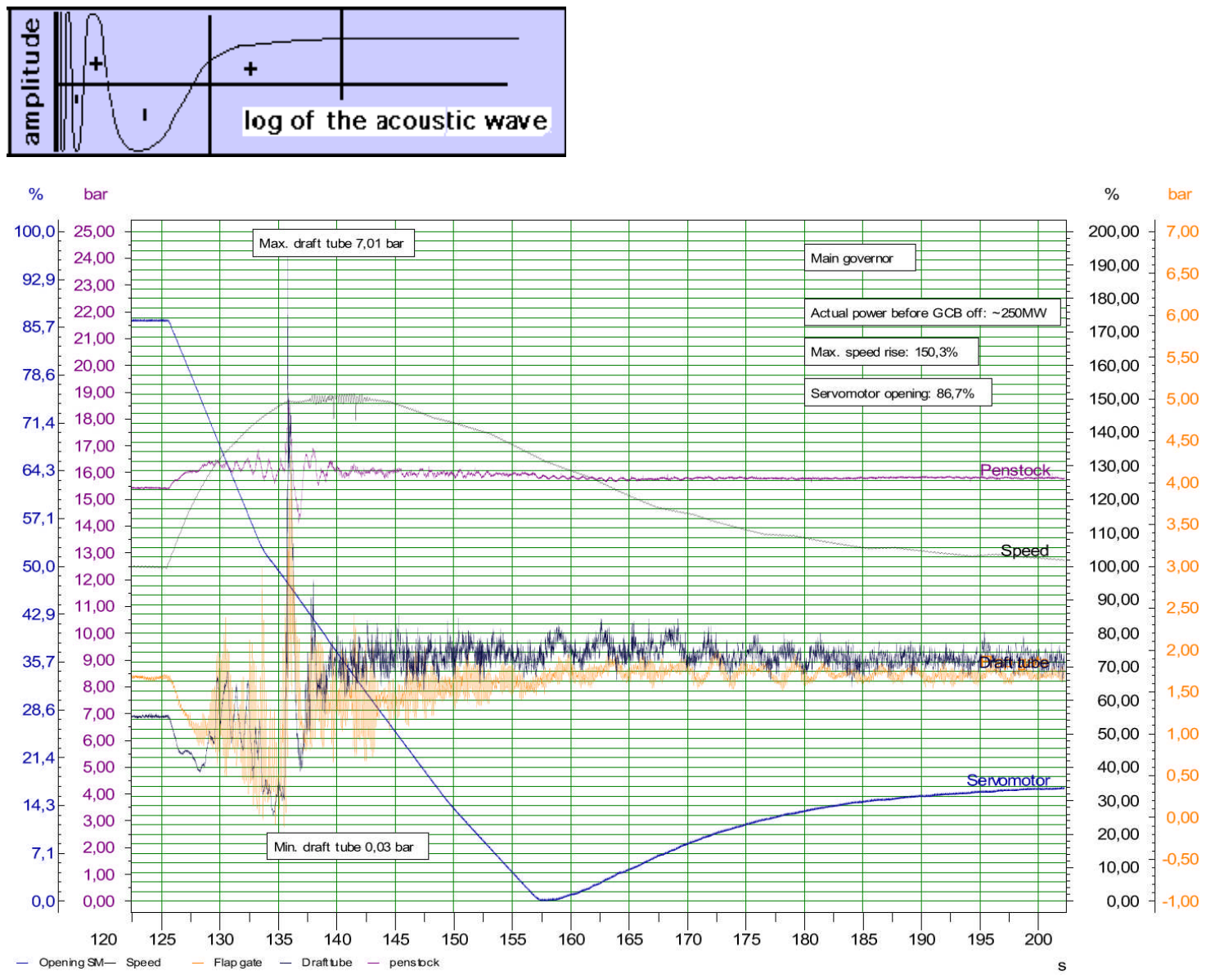

Figure 1. A severe surge during an emergency shut down 
The measurement equipment in this experiment has a sampling time of $0.01 \mathrm{sec}$. Pressure transducers measuring -1.0 to 10 Bar located at the following parts:

a. two at draft tube cone

b. one at draft tube exit

c. one at head cover

Also one pressure transducer measuring -1.0 to 25.0 Bar located at the spiral case.

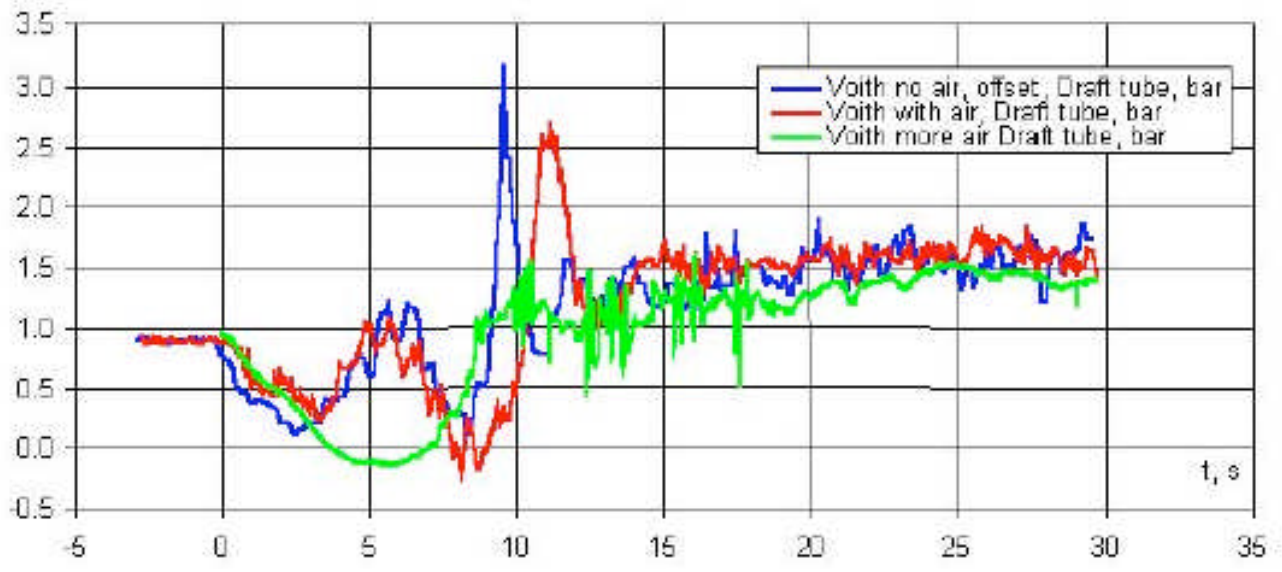

Figure 2. The decreasing of the draft tube pressure peak upon load rejection with the increase air quantity

The flow in the draft tube is complex because the turbine often operates outside its best efficiency point. The first and best-known surging problem is caused by the helical vortex cavity that causes pressure fluctuations in the range of $50 \%$ to $70 \%$ of the output at the best efficiency point. The draft tube cavity is filled with water vapor, and air if the turbine has provision for air admission in the center of runner. Air admission was proposed as a method of damping or suppressing the surge, as shown in Figure 2.

The frequency of this partial load pressure fluctuation is often referred to as the "Rheingans frequency", and it is approximately one third of the rotational speed of machine. The runner and draft tube design as well as operating conditions influence the frequency and amplitude of fluctuations. Currently available theoretical approaches are not able to model this phenomenon accurately.

In turbine model tests with provision for the observation of draft tube flow at the partial load conditions, the cavity is visible at low sigma values (low suction pressure) as a helical void rotating in the same direction as the runner (Figure 3).

For operation at full power, the helical cavity evolves into an axially symmetrical cavity with rotation in the direction opposite to the runner rotation. This may be accompanied by pressure fluctuations and possible auto-excitation.

\section{Violent TCB Jet Plasma}

The TCB jet implant is formed when phenomena like load rejection happens, and it creates a significant cavity that collapses more violently than the stable cavitation bubbles; therefore, higher energy density in the bubble contents is produced. 
The implanted jet plasma is a high-density fluctuating energy plasma, and we expect this energy plasma to increase. The high-density plasma is produced by the plasma jet, which is pinched by the changing magnetic forces that are produced through the high velocity plasma electrons. The TCB "jet" is stabilized by this pinch effect.

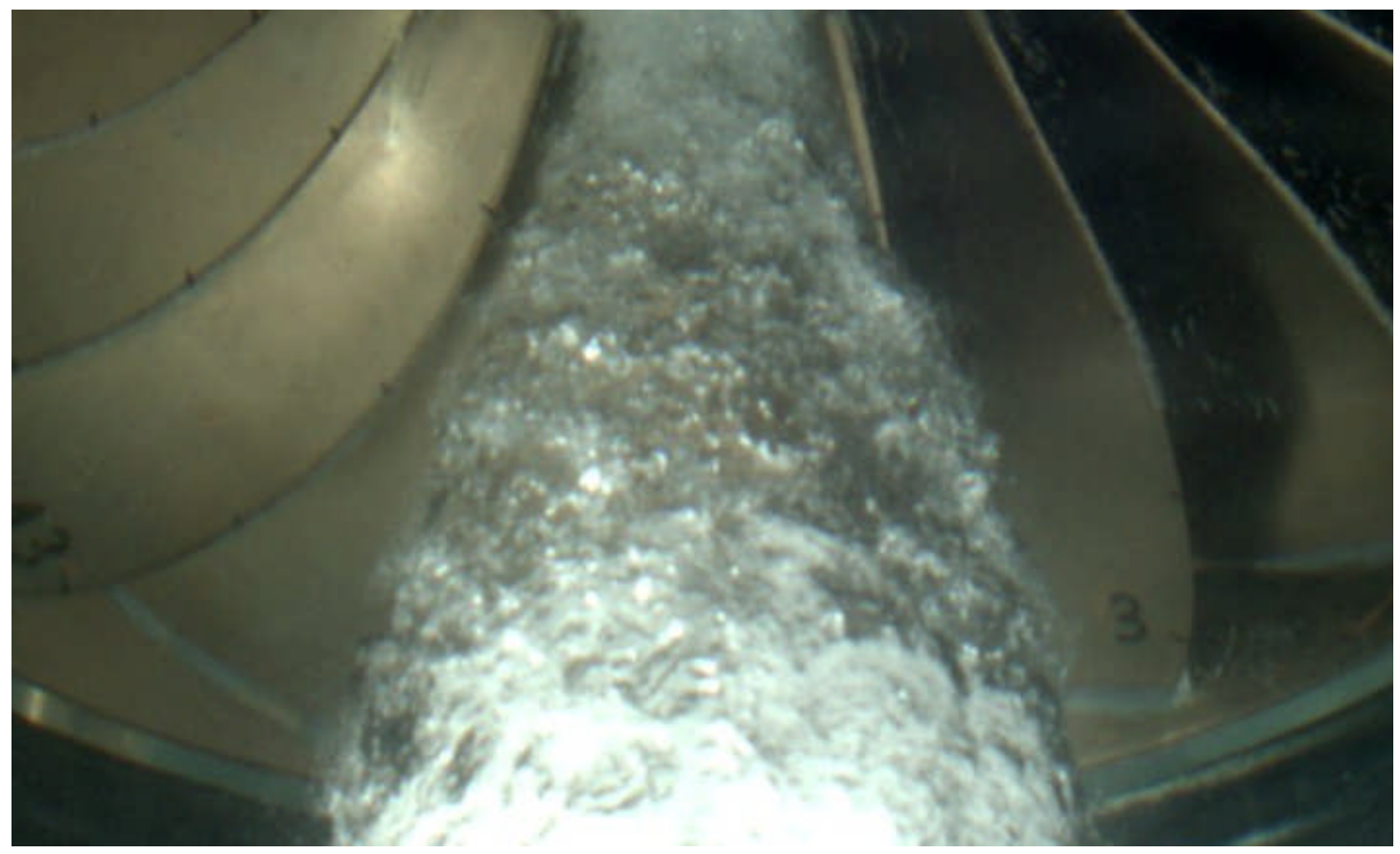

Figure 3. Cavity under runner in Model Test

The jet plasma high density changing energy contains the deuterons from the dissociated $\mathrm{D}_{2} \mathrm{O}$ that are implanted in the target lattice, and that remain in place long enough, a few picoseconds, to produce transient fusion conditions before diffusion makes it impossible for the deuterons to fuse.[1,2]

\section{Cavity Model}

The natural frequency cavity of draft tube vibrating system with cavitation region is expressed as the following relationship:[3]

$$
f_{S}=\frac{1}{2 \pi} \sqrt{\frac{A_{o} \cdot P_{c}}{\rho \cdot L_{e q} \cdot V_{c}}}
$$

Nomenclature is listed at the end of the paper. All parameters except the cavitation volume $\mathrm{Vc}$ in above equation can be determined. Observation of the cavitation vortex rope in a draft tube indicates that its radius decreases gradually from the inlet to the elbow. In operating condition where a single, cavitation vortex rope is observed, we see the root of the rope usually locates at the center in the inlet section of a draft tube. Thus, 
the flow field can be regarded as axisymmetric at the inlet. The distribution of circumferential velocity is assumed to be that of the Rankine vortex. Then, the velocity and static pressure distributions are expressed as follows:

- Force vortex region: $r_{c} \leq r \leq r_{v}$

$$
\begin{aligned}
v u & =\frac{r \Gamma}{2 \pi r^{2}} \\
p & =\frac{p \Gamma^{2}\left(r^{2}-r_{c}^{2}\right)}{8 \pi^{2} r_{v}^{4}}+P_{c}
\end{aligned}
$$

- Free vortex region: $r_{v}<r \leq R$

$$
\begin{aligned}
V u & =\frac{\Gamma}{2 \pi r} \\
p & =\frac{p \Gamma^{2}}{8 \pi^{2}}\left(\frac{1}{r_{v}^{2}}-\frac{1}{r^{2}}\right)+\frac{p \Gamma^{2}\left(r_{v}^{2}-r_{c}^{2}\right)}{8 \pi^{2} r_{v}^{4}}+p_{c}
\end{aligned}
$$

As a representative averaged pressure at the inlet section is required for determining the radius of cavitation rope $r_{c}$, we use the area-mean pressure recovery coefficient defined as follows:

$$
C p_{a}=\frac{p_{0}-p g h-\bar{p}_{a i}}{\rho\left(u_{i}^{2}+V u_{R i}^{2}\right) / 2}
$$

Where

$$
\begin{aligned}
u_{i} & =Q /\left(\pi R^{2}\right) \\
V u_{R i} & =\Gamma /(2 \pi R) \\
\bar{p}_{a i} & =\frac{p \Gamma^{2} r_{c}^{2}}{8 \pi^{2} r_{v}^{4}}\left(\frac{r_{c}^{2}}{2 R^{2}}-1\right)+\frac{p \Gamma^{2}}{\pi^{2}}\left(\frac{1}{4 r_{v}^{2}}-\frac{3}{16 R^{2}}-\frac{1}{4 R^{2}} \operatorname{In} \frac{R}{r}\right)+p_{c}
\end{aligned}
$$


Substituting eq. (9) into eq.(26), and using the following equations:

$K_{1}=\frac{H_{a}-H_{s}-H_{v}}{u_{1}^{2} / 2}$

$\frac{p_{0}}{p g}=h+H_{a}-H_{S}$

we have

$$
\frac{r_{c}^{4}}{8 r_{v}^{4}}-\frac{R^{2} r_{c}^{2}}{4 r_{v}^{4}}+\left(\frac{R^{2}}{2 r_{v}^{2}}-\frac{3}{8}-\frac{1}{2} \operatorname{In} \frac{R}{r_{v}}\right)-K_{i}\left(\frac{Q}{\Gamma R}\right)^{2}+C p_{a}\left[\left(\frac{Q}{\Gamma R}\right)^{2}+\frac{1}{4}\right]=0
$$

In violent bubble collapses, as observed in caviting flows, the bubbles can break up into many smaller fragments. Following the recent work of Brennen, depending on either Reyleigh-Taylor instability or micro-jet formation mechanisms, a simple bubble fission model is introduced to explore the rebound structure after fission and the energy dissipatedin the process.[4]

As mentioned a bubble fission model to describe the rebound structure of the fission fragments, thereby, the energy dissipated due to bubble fission, using a modified Rayleigh-Plesset equation. It is difficult to determine the number of product bubbles that would come out following bubble fission.

$$
\begin{aligned}
& R \ddot{R}+\frac{3}{2}(\dot{R})^{2}+\frac{\sigma}{2}\left[1-R^{-3 k}\right]+\frac{4}{(\operatorname{Re})} \frac{\dot{R}}{R}+\frac{2}{(W e)}\left[R^{-1}-R^{-3 k}\right]+\frac{1}{2} C_{p}=o \\
& C_{p}=\frac{\left(p^{\prime}-p_{0}^{\prime}\right)}{\frac{1}{2} p_{L}^{\prime} U^{\prime 2}} \\
& \operatorname{Re}=\frac{P_{L}^{\prime} U R_{0}^{\prime}}{\mu_{E}^{\prime}}
\end{aligned}
$$

and

$W e=\frac{p_{L}^{\prime} U^{\prime 2} R_{0}^{\prime}}{S^{\prime}}$ 
According to equation 12, during load rejection test, the volume of the cavity is highly dependent on the speed and position of wicket gates, and the rate of change of wicket gate position causes an initial pressure drop for cavity surroundings followed by an increase in that pressure. Therefore, cavity bubbles are affected according to equation 13, breaking into smaller fragments. In the transition time, figure 1 shows that the energy dissipated due to fission when the number of fission products is large. This process generates many tiny bubbles at the micro or nano levels, which is one of the characteristics of hydro turbines.

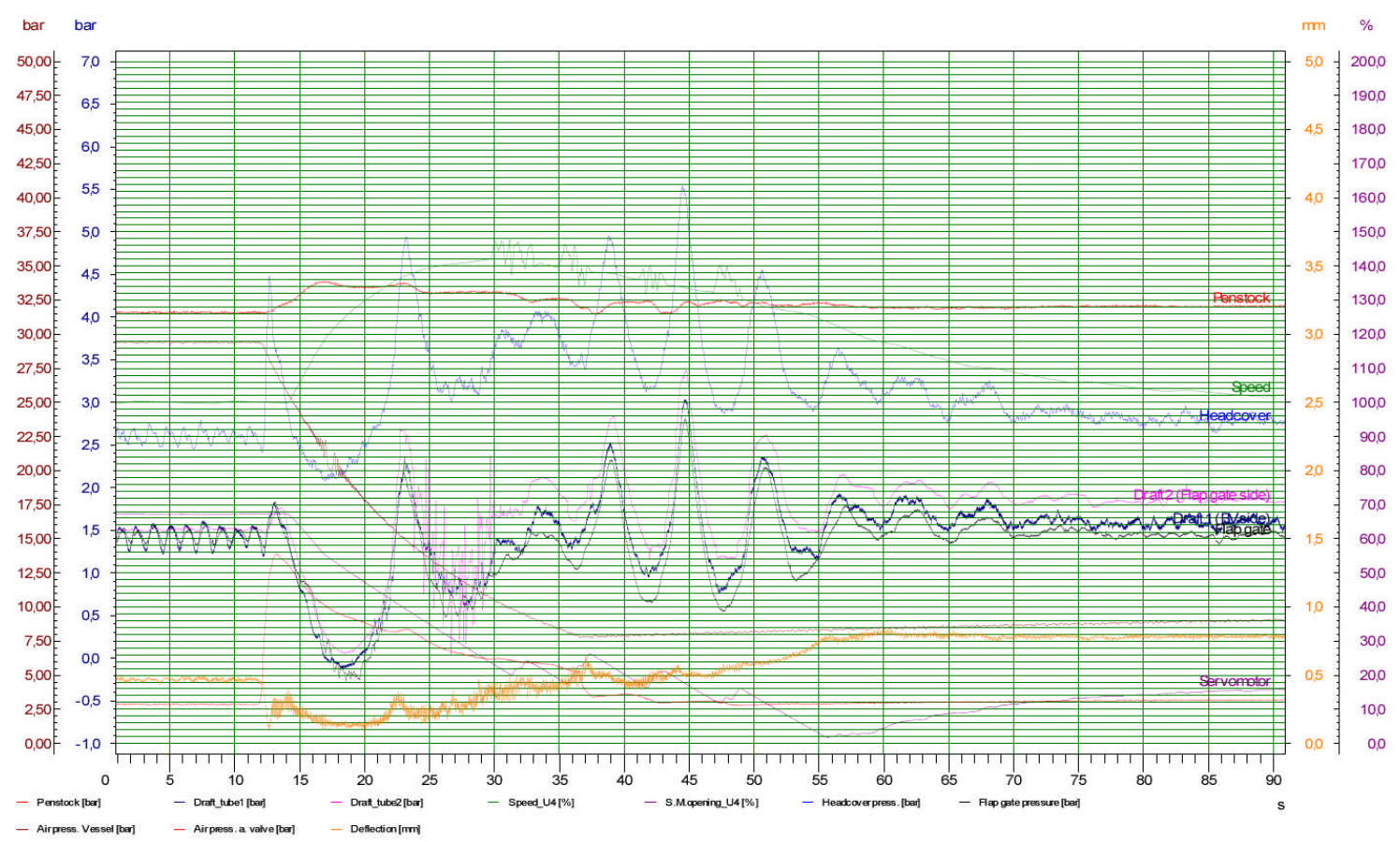

Figure 4. Several examples of TCB jet plasma generation by opening and closing of the wicket gates

\section{Conclusion}

A helical cavity, which is formed in hydro power plants during load rejection, can collapse, and violent TCB jet plasma can be implanted. It appears possible to simulate these phenomena (Load Rejection). Its energy is achievable and controllable. The level of collapse energy and frequency is controllable by the use of air injection. It is possible to generate TCB jet plasma when the wicket gates open and close several times during load rejection.

\section{Acknowledgment}

The author would like to thank J. Rothwell from LENR-CANR.org for his editorial and technical comments. 


\section{Nomenclature}

$\Gamma \quad:$ circulation

$P$ : pressure

$r_{v}:$ cove radius of Rankine vortex

$r_{c}$ : radius of cavitation rope

$R$ : radius of pipe well

$Q$ : flow rate

$r$ : radial position

$\bar{P}_{a i}$ : area mean static pressure at inlet section

$P_{0}$ : static pressure at the exit of draft tube.

$C_{p a}$ : area -mean pressure recovery coefficient

$\rho$ : density

$H_{u}$ : vapor pressure

$H_{s}:$ Suction Head

$H_{a}:$ atmospheric pressure
$C_{p}$ : pressure coefficient

$R$ : dimensionless bubble radius

$R_{0}^{\prime}$ : initial equilibrium bubble radius

Re: Reynolds number

We : Weber number

$U^{\prime}$ : characteristic speed for normalization.

$K$ : polytropic index

$p_{0}^{\prime}:$ initial liquid pressure at equilibrium

$p_{v}^{\prime}:$ saturated vapor pressure at liquid temperature.

$p_{L}^{\prime}$ : liquid density

$\sigma \quad$ : cavitation number

$v_{\text {. }}$ : cavitation volume

$L_{e q}$ : equivalent length of foot portion

$P_{c}:$ inner Pressure of cavity

$A_{0}:$ sectional area of draft tube exit

\section{References}

1. Stringham, R., Cavitation and Fusion in Tenth International Conference on Cold Fusion, 2003.

2. Stringham, R. Pinched cavitation jets and fusion events. in The 9th International Conference on Cold Fusion, Condensed Matter Nuclear Science. 2002. Tsinghua Univ., Beijing, China: Tsinghua Univ. Press

3. Wang, X., Nishi, M., and Tsukamoto, H.. A simple model for predicting the draft tube surge. in XVII IAHR Symposium, Section on Hydraulic Machinery and Cavitation. 1994. Beijing.

4. Tunc, M., and Delale, F., Energy Dissipation due to Fission of Cavitating Bubbles. In Fifth International Symposium on Cavitation, Osaka, Japn, November 1-4, 2003. 